\title{
Effect of Optical Coherence Tomography Patterns on One-year Outcomes of Aflibercept Therapy for Diabetic Macular Edema
}

\author{
Sehnaz Ozcaliskan, Sevcan Balci, Bugra Karasu and Ozgur Artunay \\ Eye Clinic, Beyoglu Eye Training and Research Hospital, University of Health Sciences, Istanbul, Turkey
}

\begin{abstract}
Objective: To evaluate the outcomes of aflibercept based on different morphologic patterns on optical coherence tomography (OCT) for diabetic macular edema (DME).

Study Design: Observational study.

Place and Duration of Study: Beyoglu Eye Training and Research Hospital, Turkey from February 2016 to March 2018. Methodology: Records of 115 eyes of 115 patients who were treated with aflibercept for DME were reviewed. Eyes were classified based on OCT features as diffuse retinal thickening (DRT, $n=37$ ), cystoid macular edema (CME, $n=40)$, and serous retinal detachment (SRD, $n=38$ ). Best-corrected visual acuity (BCVA) and central subfield retinal thickness (CST) at baseline and after three doses of aflibercept at months 3,6,12 were recorded. Primary outcome measures were the changes in BCVA and CST at follow-up visits compared with baseline.

Results: At month 3, mean BCVA was improved in all groups. Although the increase in BCVA at month 3 was significant in DRT and CME groups, it was not significant in SRD group ( $p=0.03, p<0.01$, and $p=0.22$, respectively). At month 12 , BCVA significantly improved compared to baseline values in DRT, CME, and SRD groups $(p=0.01, p=0.01$ and $p<0.01$, respectively). There was no significant difference between groups in terms of BCVA at baseline and months 3, 6, 12 $(p=0.13, p=0.67, p=0.54$, and $p=0.28$, respectively). Mean CST significantly decreased compared to baseline values at the end of month 12 (all $p<0.01$ ).

Conclusion: Aflibercept therapy provided an improvement in visual acuity and decreased CST in different morphological DME types for one year. The number of injections that could achieve this efficacy was least in DRT group, and more injections were required in SRD group.
\end{abstract}

Key Words: Aflibercept, Diabetic macular edema, Optical coherence tomography, Morphology, Pattern.

How to cite this article: Ozcaliskan S, Balci S, Karasu B, Artunay O. Effect of optical coherence tomography patterns on one-year outcomes of aflibercept therapy for diabetic macular edema. J Coll Physicians Surg Pak 2020; 30(2):149-153.

\section{INTRODUCTION}

Despite advancements in treatment modalities, diabetic macular edema (DME) is still a significant cause of visual impairment in patients with diabetes. Optical coherence tomography (OCT) is the preferred technique to monitor DME and evaluate the disease progression and treatment response. It also contributes to understanding anatomic retinal changes in DME. Based on OCT images, DME is classified into three basic types as follows: Diffuse retinal thickening (DRT), cystoid macular edema (CME), and serous retinal detachment (SRD). ${ }^{1}$ Clinical studies have shown that inflammatory and angiogenic cytokine levels in aqueous humor and vitreous may differ between these DME patterns ${ }^{2,3}$

Correspondence to: Sehnaz Ozcaliskan, Eye Clinic, Beyoglu Eye Training and Research Hospital, University of Health Sciences, Istanbul, Turkey

E-mail:drsehnaz@yahoo.com

Received: October 07, 2019; Revised: December 09, 2019;

Accepted: January 13, 2020
Vascular endothelial growth factor (VEGF) is the main cytokine that promotes angiogenesis and vascular hyperpermeability with an inflammatory response in DME. ${ }^{4}$ Pharmacological blockade of VEGF is the main therapy and pro-re-nata (PRN) protocol is the frequently preferred treatment modality. In a guideline for DME treatment, it is emphasised that OCT biomarkers may play a critical role in establishing personalised PRN scheme. ${ }^{5}$ Thus, it is essential to interpret the OCT images precisely in DME. Recently, clinicians have shown interest in artificial intelligence and computerassisted diagnosis to ensure a uniform interpretation of OCT. 6,7

In DME, tomographic features are related to visual acuity, disease progression and treatment response..$^{1,8}$ Previous studies evaluated the efficacy of ranibizumab, bevacizumab, dexamethasone in different morphologic DME types; and outcomes are varied. ${ }^{9-11}$

The aim of this study was to evaluate the anatomic and visual outcomes in patients with three main morphologic patterns of DME treated with aflibercept. 


\section{METHODOLOGY}

This study included 115 eyes of 115 patients diagnosed with DME at Beyoglu Eye Training and Research Hospital, Turkey from February 2016 to March 2018. The study was approved by the Hamidiye Ethics Committee, University of Health Sciences, and conducted in adherence to the tenets of the Helsinki Declaration. The inclusion criteria were: age of 18 years or more, Type 1 or Type 2 diabetes mellitus with non-proliferative diabetic retinopathy, a best-corrected visual acuity (BCVA) between 20/25 and 20/400 Snellen equivalent, and macular edema with a CST of at least $300 \mu \mathrm{m}$.

Exclusion criteria were: proliferative diabetic retinopathy, uncontrolled glaucoma, and ocular hypertension, any other ocular disease apart from diabetic retinopathy that may cause macular edema, focal/grid laser photocoagulation or treatment with another antiangiogenic agent within three months before first loading dose of aflibercept, history of vitrectomy, evidence of vitreomacular traction, and previous treatment with intraocular steroids within six months.

Patient charts were reviewed for demographic data and previous treatment history for DME. All patients underwent a complete ophthalmologic examination including BCVA with Snellen chart, slit-lamp anterior segment assessment, intraocular pressure measurement, fundus biomicroscopy at baseline, and follow-ups. Fluorescein angiography was performed at baseline and reevaluated, if necessary. OCT imaging was performed with Spectralis (Heidelberg Engineering, Heidelberg, Germany) at baseline and follow-up visits. According to baseline OCT scans, macular edema morphology was classified by a single observer, and the study group divided into three subgroups as diffuse retinal thickening (DRT), cystoid macular edema (CME), and serous retinal detachment (SRD). In cases where DRT is combined with CME, the pattern is classified as CME. In cases with SRD, regardless of whether they were combined with another pattern, were classified as SRD type.

Patients were treated with three consecutive $2.0 \mathrm{mg}$ intravitreal aflibercept injections at one-month intervals initially. Patients then had monthly follow-up and treated on an as-needed basis. Retreatment with aflibercept was evaluated, if macular edema persisted; and BCVA worsened in comparison with the preceding visit. Measurements at baseline and months 3, 6, 12 were recorded. Primary outcome measures were the changes in BCVA and CST at follow-up visits compared with baseline. For statistical analysis, BCVA scores were converted into logMAR notation.

Statistical analysis was performed using Statistical Package for Social Sciences (SPSS) for Windows 23.0 programme. The normality of the data was analysed using the Kolmogorov-Smirnov test. Descriptive statistics and one-factor analysis of variance (ANOVA) were used to describe the demographic data of the study groups. The differences in mean BCVA and CST at baseline and follow-up periods were analysed with paired t-test in each group. The mean parameter of BCVA and CST values in three groups was compared using the onefactor ANOVA, and differences among groups were assessed using the Bonferroni post-hoc test. A p-value of $<0.05$ was considered as statistically significant.

\section{RESULTS}

The study group consists of 115 eyes of 115 patients with a mean age of $63.80 \pm 7.74$ years. There were 70 $(60.9 \%)$ men and 45 (39.1\%) women in the study group. There were 37 eyes in the DRT group, 40 eyes in the CME group, and 38 eyes in the SRD group. Baseline characteristics of patients and eyes among three different groups are shown in Table I.

The baseline mean BCVA was $0.46 \pm 0.35 \log M A R$ in the DRT group, $0.47 \pm 0.21 \log M A R$ in the CME group, and $0.59 \pm 0.34 \log M A R$ in the SRD group $(p=0.13)$. After three loading doses of aflibercept at month 3 , the mean BCVA was improved to $0.40 \pm 0.33$ logMAR, $0.39 \pm 0.22$ logMAR, and $0.53 \pm 0.34 \log M A R$ in DRT, CME and SRD groups respectively. Although the increase in BCVA at month 3 was significant in DRT and CME groups, it was not significant in the SRD group. $(p=0.03, p<0.001$, and $p=0.22$, respectively). At months 6 and 12, a significant improvement was observed in BCVA compared with baseline values in CME and SRD groups. $(p=0.01$ and, $p=0.03$ respectively). At month 12, BCVA was significantly improved in all groups compared to baseline values (all, $p=0.01$, Figure 1). There was no significant difference between three groups in terms of BCVA at baseline and months $3,6,12 .(p=0.13, p=0.67, p=0.54$, and $p=0.28$, respectively).

At baseline, mean CST was $403.46 \pm 77.77 \mu \mathrm{m}$ in the DRT group, $448.50 \pm 110.05 \mu \mathrm{m}$ in the CME group and $516.97 \pm 149.18 \mu \mathrm{m}$ in the SRD group. Baseline mean

Table I: Baseline characteristics of patients and eyes among three different optic coherence tomography patterns of diabetic macular edema.

\begin{tabular}{|c|c|c|c|c|}
\hline & $\begin{array}{c}\text { All } \\
n=115\end{array}$ & $\begin{array}{c}\text { DRT } \\
\mathrm{n}=37\end{array}$ & $\begin{array}{l}\text { CME } \\
n=40\end{array}$ & $\begin{array}{l}\text { SRD } \\
n=38\end{array}$ \\
\hline Gender (male/female) & $70 / 45$ & $23 / 14$ & $22 / 18$ & $25 / 13$ \\
\hline Mean age* & $63.80 \pm 7.74$ & $64.10 \pm 7.73$ & $64.92 \pm 6.97$ & $62.31 \pm 8.46$ \\
\hline Eye (right/left) & $54 / 61$ & $21 / 16$ & $18 / 22$ & $15 / 23$ \\
\hline \multicolumn{5}{|l|}{$\begin{array}{l}\text { Previous treatment history } \\
\text { (eyes) } \%\end{array}$} \\
\hline $\begin{array}{l}\text { Panretinal laser Photo- } \\
\text { coagulation }\end{array}$ & $40 / 115(34.8)$ & 14/37 (37.8) & $14 / 40(35.0)$ & 12/38 (31.6) \\
\hline Focal laser & $11 / 115(9.5)$ & $2 / 37(5.4)$ & $3 / 40(7.5)$ & $3 / 38(7.9)$ \\
\hline Intravitreal anti-VEGF & $45 / 115(39.1)$ & $12 / 37(32.4)$ & $20 / 40(50.0)$ & $13 / 38(34.2)$ \\
\hline $\begin{array}{l}\text { Intravitreal dexametha- } \\
\text { sone }\end{array}$ & $15 / 115(13.0)$ & $6 / 37(16.2)$ & $7 / 40(17.5)$ & 2/38 (5.3) \\
\hline
\end{tabular}

$\overline{D R T}=$ Diffuse retinal thickness; $C M E$ = Cystoid macular edema; $S R D=$ Serous retinal detachment; DME: Diabetic macular edema; PRP: Panretinal photocoagulation. ${ }^{*}$ Not significant $(p>0.05)$ by one-factor analysis of variance (ANOVA). 
CST values in the DRT and CME groups were significantly lower than in the SRD group $(p<0.001$, $p=0.032$ ). At month 3 , there was a significant difference in mean CST between CME and SRD groups $(p=0.037)$. At months 3, 6, and 12, a significant reduction in CST in all groups compared with baseline values were observed (all, $p<0.01$ ) (Figure 2). There was no significant difference between three groups in terms of CST at months 6 and 12 ( $p=0.299, p=0.165$, respectively).

At the end of month 12, the mean number of injections in the DME group was $4.86 \pm 1.16$, in CME group 5.17 \pm 1.41 , and in SRD group $5.34 \pm 1.49$. There was no difference in the number of injections based on DME types $(p=0.311)$.

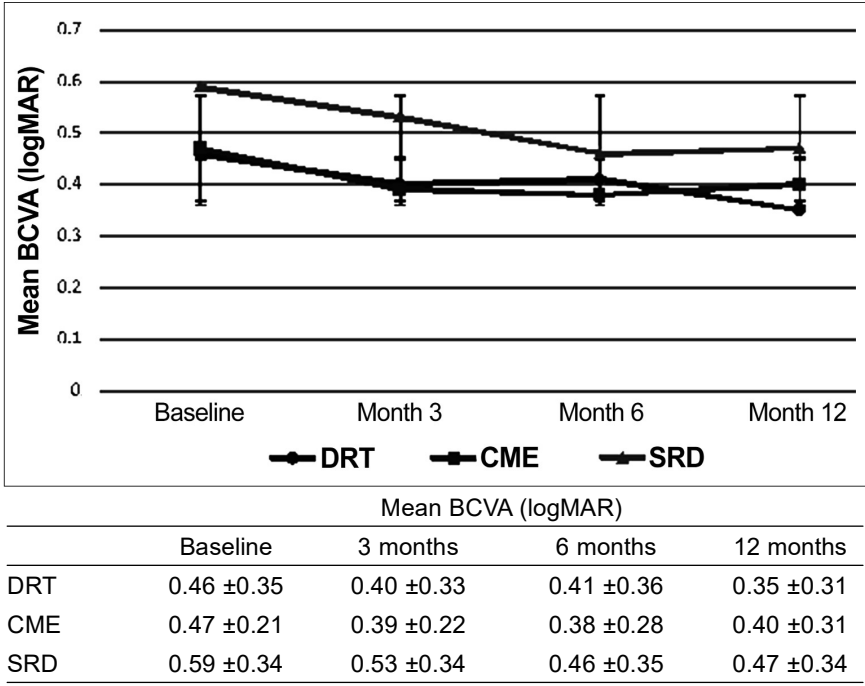

Figure 1: Changes in mean best-corrected visual acuity (BCVA) over time in groups. Error bar indicates standard deviation.

$D R T=$ Diffuse retinal thickening; $C M E=$ Cystoid macular edema; $S R D=$ Serous retinal detachment.

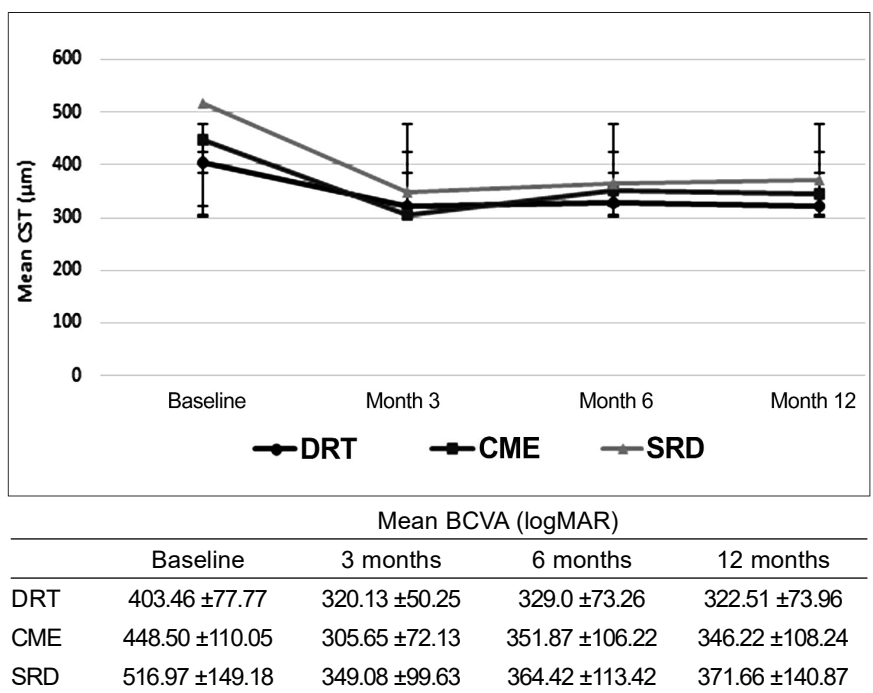

Figure 2: Changes in mean central subfield retinal thickness (CST) over time in groups. Error bar indicates standard deviation.

DRT: Diffuse retinal thickening, CME: Cystoid macular edema, SRD: Serous retinal detachment.

\section{DISCUSSION}

Diabetic macular edema is one of the important public health problems worldwide with its high prevalence and socioeconomic aspect. Recent randomised clinical trials have shown that anti-VEGF treatment improves visual acuity and provides regression of macular edema. ${ }^{12-14}$ Local studies evaluating anti-VEGF agents in DME also demonstrated favourable visual and anatomic outcomes with bevacizumab, ranibizumab, and aflibercept. 15,16

Intravitreal anti-VEGF treatment requires repeated injections to maintain the therapeutic effect. Repeated injections are associated with increased financial burden as well as systemic and ocular safety issues. Thus, individual treatment approaches are needed to reduce the injection frequency. OCT biomarkers give clues to the progression of the disease and treatment response. In this study, with aflibercept, improved visual acuity and decreased CST for 12 months in three different morphologic DME patterns were observed significantly

In the present study, at the baseline, the mean BCVA was lower and mean CST was significantly thicker in the SRD group than the DRT and CME groups. In a study evaluating DME patterns and related risk factors, the CME group showed the highest CST and the worst visual acuity. 17 Similar to these findings, Alkuraya et al. reported that sponge-like retinal swelling type had the best baseline visual acuity and the least CST values. Presence of SRD is admitted as a sign of disease activity and thought to have a negative effect on visual functions. The poor prognosis is most likely related to the disruption of ELM.18 After three doses of aflibercept, improvement in BCVA was not significant in the SRD group. However, CST improvement was significant in all three groups. The difference between groups may be related to the ELM damage and associated functional alterations.

In DRT, anchor proteins of endothelial tight junctions damage, and consecutively inner blood-retinal barrier disrupts vascular permeability increases. ${ }^{19}$ The development of SRD type compared to CME and DRT type macular edema is not yet fully elucidated. Disruption of ELM may lead the accumulated fluid in the outer retina to move into the subretinal space. Higher levels of IL-6 in SRD type compared to DRT and CME also suggest that inflammation has an important role in pathogenesis. ${ }^{20}$ The relationship between SRD and visual functions in diabetic macular edema is controversial. A post-hoc analysis of RISE and RIDE trials showed that the presence of baseline subretinal fluid was predictive for better visual outcomes than those without baseline SRD.21 Korobelnik et al. evaluated the potential protective role of SRD by a post-hoc analysis of the VIVID and VISTA studies.22 They demonstrated that BCVA gain with aflibercept was similar between groups with and without SRD at baseline. The efficacy of 
aflibercept versus laser therapy seemed to be greater in the group with SRD at baseline. Shimura et al. evaluated the efficacy of single-dose bevacizumab in terms of edema morphology in DME. 8 They showed the effect of bevacizumab as greatest, intermediate, and weakest in the DRT, CME, and SRD group, respectively. They also concluded that VEGF might play a critical role in the pathogenesis of spongiform and cystoid edema compared to SRD. 8

In this study, we observed significantly improved visual acuity and decreased CST for 12 months with aflibercept in different morphologic DME patterns. The mean number of injections was fewer in the DRT with 4.86 compared to 5.17 in CME group and 5.34 in SRD group. However, the difference between groups was not significant. Seo et al. also reported the mean number of injections to be the lowest in the DRT group in their prospective study. ${ }^{9}$ They related this outcome to the early diagnosis of DRT, which defined as CST increase without cystic changes and subretinal fluid. Diffuse retinal thickening can be considered as a morphological type that occurs before CME and SRD development. To demonstrate this, studies evaluating the changes in OCT morphology over time should be designed.

Aflibercept therapy provided improvement in visual acuity and decrease in subfield thickness in different morphological DME types for one year. The number of injections that could obtain this efficacy was least in the DRT type, whereas more injections were required in SRD type. In addition to further studies that will elucidate the pathophysiology of SRD, prospective cohort studies with different agents and treatment protocols are required to determine new therapeutic approaches.

\section{CONCLUSION}

Aflibercept therapy provides improvement in visual acuity and decrease in subfield thickness in different morphological DME types for one year. The number of injections that can obtain this efficacy is least in the DRT type, whereas more injections are required in SRD type. In addition to further studies that will elucidate the pathophysiology of SRD, prospective cohort studies with different agents and treatment protocols are required to determine new therapeutic approaches.

\section{ETHICAL APPROVAL:}

Hamidiye Ethics Committee, University of Health Sciences, approved this study, and ethical approvals were obtained prior to initiation of the research work.

\section{PATIENTS' CONSENT:}

As the study was conducted retrospectively, data was obtained from clinical archive after obtaining necessary permissions from hospital management.

\section{CONFLICT OF INTEREST:}

Authors declared no conflict of interest.

\section{AUTHORS' CONTRIBUTION:}

SO: Conducted data collection, drafting, analysis, and interpretation of data and manuscript writing.

SB: Helped in writing the manuscript.

BK: Commented on an earlier version of the manuscript. OA: Revised the manuscript critically for important intellectual content; approved the final version to be published.

\section{REFERENCES}

1. Otani T, Kishi S, Maruyama Y. Patterns of diabetic macular edema with optical coherence tomography. Am J Ophthalmol 1999; 127:688-93.

2. Moosang K, Yonguk K, Lee SJ. Comparison of aqueous concentrations of angiogenic and inflammatory cytokines based on optical coherence tomography patterns of diabetic macular edema. Indian J Ophthalmol 2015; 63:312-7.

3. Sonoda S, Sakamoto T, Yamashita T, Shirasawa M, Otsuka H, Sonoda Y. Retinal morphologic changes and concentrations of cytokines in eyes with diabetic macular edema. Retina 2014; 34:741-8.

4. Behl T, Kotwani A. Exploring the various aspects of the pathological role of vascular endothelial growth factor (VEGF) in diabetic retinopathy. Pharmacol Res 2015; 99:137-48.

5. Schmidt-Erfurth U, Garcia-Arumi J, Bandello F, Berg K, Chakravarthy U, Gerendas BS, et al. Guidelines for the management of diabetic macular edema by the european society of retina specialists (euretlna). Ophthalmologica 2017; 237:185-222.

6. Ting DSW, Pasquale LR, Peng LR, Pasquale, Peng L, Peter J. Artificial intelligence and deep learning in ophthalmology. $\mathrm{Br} \mathrm{J}$ Ophthalmol 2019; 103:167-75.

7. Kapoor R, Whigham BT, Al-Aswad LA. Artificial intelligence and optical coherence tomography imaging. Asia Pac J Ophthalmol (Phila) 2019; 8:187-94.

8. Yamamoto S, Yamamoto T, Hayashi M, Takeuchi S. Morphological and functional analyses of diabetic macular edema by optical coherence tomography and multifocal electroretinograms. Graefes Arch Clin Exp Ophthalmol 2001; 239:96-101.

9. Shimura M, Yasuda K, Yasuda M, Nakazawa T. Visual outcome after intravitreal bevacizumab depends on the optical coherence tomographic patterns of patients with diffuse diabetic macular edema. Retina 2013; 33:740-7.

10. Seo KH, Yu SY, Kim M, Kwak HW. Visual and morphologic outcomes of intravitreal ranibizumab for diabetic macular edema based on optical coherence tomography patterns. Retina 2016; 36:588-95.

11. Figueras-Roca M, Sala-Puigdollers A, Zarranz-Ventura J, Alba-Linero C, Alforja S, Esquinas C, et al. Anatomic response to intravitreal dexamethasone Implant and baseline aqueous humor cytokine levels in diabetic macular edema. Invest Ophthalmol Vis Sci 2019; 60:1336-43.

12. Mitchell P, Bandello F, Schmidt-Erfurth U, Lang GE, Massin P, Schlingemann RO, et al. The restore study: Ranibizumab monotherapy or combined with laser versus laser mono- 
therapy for diabetic macular edema. Ophthalmology 2011; 118:615-25.

13. Brown DM, Schmidt-Erfurth U, Do DV, Holz FG, Boyer DS, Midena $\mathrm{E}$, et al. Intravitreal aflibercept for diabetic macular edema: 100-week results from the vlsta and vlvld studies. Ophthalmology 2015; 122:2044-52.

14. Diabetic retinopathy clinical research network, Wells JA, Glassman AR, Ayala AR, Jampol LM, Aiello LP, Antoszyk AN, et al. Aflibercept, bevacizumab or ranibizumab for diabetic macular edema. N Engl J Med 2015; 372:1193-203.

15. Uslu C, Gülkilik GI, Oba E, Aslan B. Intravitreal bevacizumab for diffuse diabetic macular edema: early results. TJO 2010; 40:145-50.

16. Alim S, Demir AK. The effect of serum hba1c level on Intravitreal anti-vegf treatment for diabetic macular edema. Turk Diyab Obez 2019; 2:79-83.

17. Ahmadpour-Baghdadabad M, Manaviat M, ShojaoddinyArdekani A. Optical coherence tomography in diabetic macular edema: patterns and related risk factors. Nepal J Ophthalmol 2013; 5:190-4.
18. Alkuraya H, Kangave D, Abu El-Asrar AM. The correlation between optical coherence tomographic features and severity of retinopathy, subfield thickness and visual acuity in diabetic macular edema. Int Ophthalmol 2005; 26:93-9.

19. Antonetti DA, Barber AJ, Khin S, Lieth E, Tarbell JM, Gardner TW, et al. Vascular permeability in experimental diabetes is associated with reduced endothelial occludin content: Vascular endothelial growth factor decreases occludin in retinal endothelial cells. Penn State Retina Research Group. Diabetes 1998; 47:1953-9.

20. Kaya M, Kaya D, Idiman E, Kocak N, Ozturk T, Ayhan Z, et al. A novel biomarker in diabetic macular edema with serous retinal detachment: serum chitinase-3-like protein 1. Ophthalmologica 2019; 241:90-7.

21. Sophie R, Lu N, Campochiaro PA. Predictors of functional and anatomic outcomes in patients with diabetic macular edema treated with ranibizumab. Ophthalmology 2015; 122:1395-401.

22. Korobelnik JF, Lu C, Katz TA, Dhoot DS, Loewenstein A, Arnold $\mathrm{J}$, et al. Effect of baseline subretinal fluid on treatment outcomes in vivid-dme and vista-dme studies. Ophthalmol Retina 2019; 3:663-9. 